\title{
Chlamydia trachomatis Testing Sensitivity in Midstream Compared With First-Void Urine Specimens
}

Derelie Mangin, $\mathrm{MB}, \mathrm{CbB}, \mathrm{DPH} \mathrm{H}^{1}$

David Murdoch, MB, CbB, MD ${ }^{2}$

J. Elisabeth Wells, $P h D^{3}$

Edward Coughlan, $M B, C b B$, FACbSHM ${ }^{4}$

Sue Bagshaw, MB, CbB

Paul Corwin, $\mathrm{MB}, \mathrm{CbB}^{6}$

Stephen Chambers, $M B, C b B, M D^{2}$

Les Toop, $M B, C b B, M D^{3}$

'Primary Care Research Unit, Department of General Practice, University of Otago,

Christchurch, New Zealand

${ }^{2}$ Department of Pathology, University of Otago, Christchurch, New Zealand

${ }^{3}$ Department of Public Health and General Practice, University of Otago, Christchurch, New Zealand

${ }^{6} \mathrm{~A}$ private practice, Christchurch, New Zealand

${ }^{4}$ Canterbury District Health Board Sexual Health Clinic, Christchurch, New Zealand

${ }^{5}$ Christchurch Youth Health Clinic, Christchurch, New Zealand

Conflicts of interest: authors report none.

\section{CORRESPONDING AUTHOR}

Dee Mangin, MB, ChB, DPH

Department of General Practice Christchurch School of Medicine and Health Sciences

PO Box 4345

Christchurch, 8140, New Zealand dee.mangin@otago.ac.nz

\begin{abstract}
PURPOSE Traditionally first-void urine specimens are used to test for Chlamydia trachomatis. In contrast, midstream urine specimens are traditionally recommended for microscopy and culture of presumptive bacterial urinary tract infections. The ability to test for both $C$ trachomatis and urinary tract infection on a single midstream urine specimen would greatly aid clinical practice, as an urinary tract infection is an extremely common complaint in primary care. This study set out to determine how well positive $C$ trachomatis results obtained on first-void specimens would correlate with positive findings in matched midstream specimens.
\end{abstract}

METHODS One hundred women with a first-void urine specimen positive for $C$ trachomatis also provided midstream specimens for comparison. All specimens had $\mathrm{C}$ trachomatis testing performed using a DNA detection method.

RESULTS Of the 100 eligible participants with a first-void specimen positive for C trachomatis, $96(96 \%)$ also had a positive midstream specimen (95\% exact confidence limits, 90.1\%, 98.9\%).

CONCLUSIONS These results suggest that by using newer nucleic acid amplification techniques (NAATs), timing of specimen collection is not so important in testing for $C$ trachomatis as previously thought. The sensitivity of NAAT testing on midstream urine specimens in women is sufficiently equivalent to testing on first-void specimens to consider in clinical practice and research settings where first-void specimens have formerly been collected.

Ann Fam Med 2012;10:50-53. doi:10.1370/afm.1323.

\section{INTRODUCTION}

C blamydia trachomatis (C trachomatis) is the most common sexually transmitted bacterial infection. ${ }^{1}$ It may have few, if any, symptoms in women. The complications of infertility, pelvic inflammatory disease, and ectopic pregnancy are serious, yet treatment is simple once infection is detected. Although there are a variety of methods for testing for $C$ trachomatis, no single test is ideal. Currently, to test for the infection in women, nucleic acid amplification tests (NAATs) are preferred, and either a vaginal swab or first-void urine specimen is recommended for case finding, but neither has a 100\% sensitivity. In the United States vaginal swabs are the preferred sample..$^{2-4}$

Dysuria and increased urinary frequency associated with presumed urinary tract infection are extremely common reasons for seeking treatment in primary care. ${ }^{5}$ About $10 \%$ of women suffer from symptoms of a urinary tract infection in any year, ${ }^{6}$ and seeking treatment provides a potential opportunity to detect both symptomatic and asymptomatic infection with C trachomatis. The sexually active woman is at risk of both urinary tract infection and $C$ trachomatis infection. 
One difficulty in testing for $C$ trachomatis in women seeking treatment for dysuria and frequency is that traditionally a first-void urine specimen has been recommended, which is different from the midstream specimen used to diagnose a urinary tract infection. This distinction is made on the basis that $C$ trachomatis in the urethra was more likely to be captured in the first urine passing over the epithelial cells, whereas bacteria from this portion of the urine stream were more likely to be skin commensals than the intravesical pathogens of cystitis.

Testing methods have improved markedly in recent years, and NAATs are more sensitive than past methods. ${ }^{7}$ If NAATs for $C$ trachomatis are sensitive enough to use on a standard midstream urine sample, it would be possible to avoid asking the patient complaining of a urinary tract infection to provide an additional firstvoid urine sample or an invasive vaginal swab test for C trachomatis. This pilot study set out to see how many positive results obtained on first-void urine specimens would be missed if a midstream specimen is used. Accordingly, we compared the accuracy of $C$ trachomatis testing using NAAT techniques on paired first-void and midstream urine specimens from women known to be infected with $C$ trachomatis, confirmed by testing a vaginal swab sample. This method allowed us to determine whether testing midstream specimens is sufficiently sensitive to be considered for routine clinical use as an alternative to testing first-void specimens.

\section{METHODS}

\section{Study Design}

This prospective study involved sample collection of both first-void and midstream urine specimens. Because the population prevalence of $C$ trachomatis infection is relatively low, to find adequate numbers of participants, the study sample was drawn from women for whom a vaginal swab specimen recently tested positive for $C$ trachomatis.

This study was approved by the Upper South Region Ethics Committee, New Zealand.

\section{Study Population}

Women attending the Canterbury District Health Board Sexual Health Clinic, Youth Health Clinic, or Family Planning Clinic in Christchurch, whose vaginal swab was positive for $C$ trachomatis by NAAT, and who were returning for treatment with antibiotics were invited to participate in the study. Nonconsenting women and women who had already received antibiotic treatment were excluded from the study. Women were asked to provide 2 urine specimens - a first-void and a midstream specimen. The results for all those with a positive vaginal swab are described in the Results section; those with a positive first-void specimen for $C$ trachomatis using the testing methods described below were included in the comparison study.

\section{Sample Size and Analysis}

The study was designed to determine the sensitivity of the NAAT on midstream specimens relative to the clinical norm of a NAAT-positive first-void specimen. All samples were swab positive. A sensitivity of $90 \%$ was chosen as the lower limit of the necessary sensitivity for consideration in a clinical or research setting. A sample size of 100 positive first-void specimens was selected to estimate the sensitivity of midstream specimens. If the true sensitivity was $95 \%$, then the probability was greater than $95 \%$ that the sample estimate would be at least $90 \% .{ }^{8}$ Exact confidence limits around the sample estimate were calculated. ${ }^{9}$

\section{Sample Collection}

Participating women provided a first-void sample of 10 to $20 \mathrm{~mL}$ (urethral) urine and a midstream (bladder) urine sample at the time they returned for their antibiotic prescription. Careful written, verbal, and pictorial instructions were given for collecting accurate specimens according to local midstream urine specimen collection guidelines. ${ }^{10}$ Paired samples were anonymous and identified using a unique identification number. Both urine samples were sent to the local hospital laboratory, Canterbury Health Laboratories, Christchurch, where the standard diagnostic laboratory testing was performed for $C$ trachomatis.

\section{Laboratory Analysis}

All specimens had C trachomatis testing performed using a DNA detection method (BD ProbeTec strand displacement amplification, Becton Dickinson, Franklin Lakes, New Jersey). Patients had their first-void and midstream urine specimens tested for $C$ trachomatis using the same technique. All specimens were tested at the same time. Data were entered into a database (Microsoft Office Access, Microsoft Corp, Redmond, Washington) and exported into SAS 9.1 statistical software (SAS Institute, Cary, North Carolina) using a Microsoft Excel spreadsheet. All data were double entered to check for accuracy. We estimated the sensitivity of testing for $C$ trachomatis on midstream specimens using positive first-void specimens as the reference standard.

\section{RESULTS}

\section{Participants}

Recruitment occurred in 2007 to 2008. One hundred fifteen patients were initially identified as potentially 
eligible for the study. Seven were excluded from the analysis because they did not meet inclusion criteria. The reasons are outlined in detail in Figure 1. Two had been treated with antibiotics before obtaining the urine specimens, 3 urine specimens were too old to test (they had been stored in a refrigerator for several days before sending to the laboratory), 1 specimen was mislabeled so matching was not possible, and 1 was received without a consent form for study participation.

Of 108 potentially eligible patients whose vaginal swab samples were positive for $C$ trachomatis, 7 (6\%) had negative results when their first-void urine was tested, and 1 had inconclusive results. All these patients also had negative midstream urine test results, leaving 100 eligible patients whose urine tested positive for $C$ trachomatis in paired samples.

\section{Demographic and Clinical Features}

The age range of participants was 17 to 45 years (median 20 years). The reason for visiting the clinics was recorded for 61 participants: 25 (41\%) had come in for a general sexual health check, 7 (11\%) had urinary tract symptoms, 14 (23\%) complained of other symptoms, and 15 (25\%) had had a partner whose test was positive for $C$ trachomatis.

\section{Comparison of First-Void and Midstream Specimen Sensitivity}

Comparing the sensitivity of first-void and midstream urine specimens, of the 100 paired specimens with a first-void specimen positive for $C$ trachomatis, 96 (96\%) also had a midstream specimen positive for $C$ trachomatis (95\% exact confidence limits, 90.1, 98.9). ${ }^{9}$

\section{DISCUSSION}

These results suggest that when using newer NAAT techniques, timing of urine specimen collection is not as important in testing for $C$ trachomatis as previously thought. This is supported by similar findings in a study that allows a small $(n=19)$ group of first-void and midstream urine samples positive for $C$ trachomatis to be compared, together with vaginal, urethral, and cervical swabs, for detection of $C$ trachomatis. ${ }^{11}$ If the results of this pilot study are confirmed in similar studies, NAAT offers the possibility that midstream urine specimens in women may be sufficiently equivalent to testing firstvoid specimens in clinical practice as a case finding tool to improve detection of $C$ trachomatis where collecting other types of samples is impracticable. It also provides a basis for prevalence estimates in research settings where midstream urine specimens are available.

There is no reference standard for $C$ trachomatis testing that gives $100 \%$ sensitivity. The study was not

\section{Figure 1. Recruitment flowchart.}

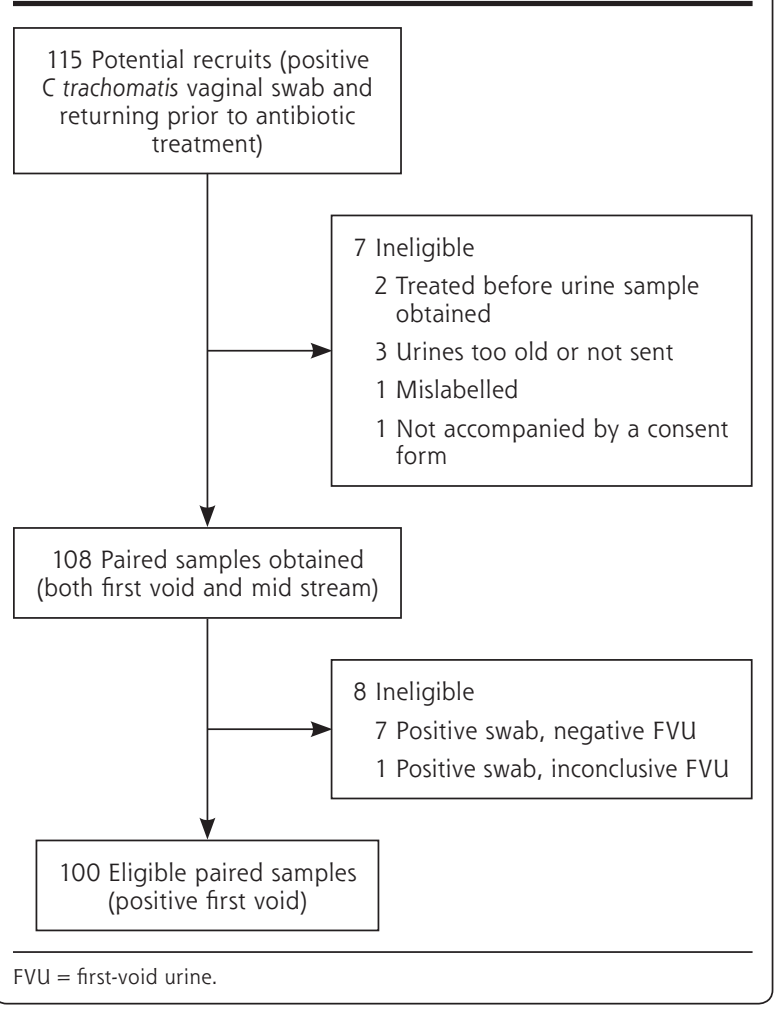

carried out on women who had symptoms of a urinary tract infection. Although this population would be ideal for the study design, because the prevalence of $C$ trachomatis infection is low, a great number of women would need to be tested, so we chose a pragmatic approach to explore the question. The study was not designed to compare the range of samples that might be used in testing for $C$ trachomatis, and the number of women whose midstream urine sample might have tested positive but whose vaginal swab test was negative is not known. Current consensus guidelines in the United States suggest that vaginal swab and cervical swab specimens are equivalent and that first-void urine specimens may be less sensitive. ${ }^{4}$ Future research might include a complete evaluation comparing the various types of specimens among a range of diagnostic tests in a population of women suspected or at-risk of having $C$ trachomatis infection. The study was also not designed to compare the tests in men, and the very low prevalence of urinary tract infections in men would limit the utility of results.

\section{Clinical Implications}

The clinical practice implications of these results are important: urine culture and $C$ trachomatis testing may be considered on a single specimen. In the clinical setting, using one sample avoids the practical difficulties of collecting (or being unable to collect) further specimens 
from patients with uncertain diagnosis after midstream urine testing for urinary tract infection. It may also be helpful where the women are at risk or report symptoms of both infections. At present, a choice must be made at the time of the consultation as to which specimen is the most important. Patients may not return to provide a second specimen or consent to invasive testing, or the diagnosis may be considered after the patient has left the clinic, eg, when a urine result showing sterile pyuria might raise the possibility of infection with $C$ trachomatis.

Assessing the impact and value of different strategies is hampered by inadequate epidemiological information on population prevalence. ${ }^{8}$ Our findings suggest that this method of testing may be suitable for use in epidemiological research for estimating the prevalence of chlamydial infection in different population groups. In epidemiological studies of antibiotic resistance in urinary tract infection, for example, the prevalence of $C$ trachomatis infections could also be estimated. ${ }^{12}$ Such information could further inform screening strategies by allowing testing for $C$ trachomatis on midstream urine specimens routinely processed by laboratories to gain initial information on whether prevalence is sufficiently high to consider targeted screening. It is worthwhile investigating and discussing further whether testing for $C$ trachomatis should be added to the usual testing panel for women's midstream urine samples sent for culture.

To read or post commentaries in response to this article, see it online at http://www.annfammed.org/content/10/1/50.

Key words: Chlamydia trachomatis; urinary tract infections

Submitted June 26, 2010; submitted, revised, June 7, 2011; accepted, July 7, 2011.

Author contributions: D. Mangin, D. Murdoch, E. Coughlan, P Corwin, and L. Toop conceived the study. D Mangin designed and coordinated the study and analysis and wrote the first draft of the paper. J. E. Wells provided biostatistical advice on design and analysis. E. Coughlan and S. Bagshaw oversaw implementation and coordination in the 2 clinics. D. Murdoch and S. Chambers advised on microbiological aspects of planning. All authors reviewed and commented on the final manuscript. D. Mangin is the guarantor.

Funding support: This study was funded by an Otago University Research Grant.
Disclaimer: Otago University had no role in the design data collection, analysis, or writing of this paper.

Preliminary results of this study were presented at the NAPCRG conference in Montreal, 2009.

Acknowledgements: This study was made possible by the staff of the Canterbury District Health Board Sexual Health Clinic, the Christchurch Youth Health Clinic, and Family Planning Clinic, Christchurch, who identified and recruited suitable patients. Toni Stewart, research nurse, provided enthusiasm, as well as ensuring smooth efficient data collection. Thanks to the Canterbury Health Limited Laboratory serology staff and particularly Sheryl Young, for managing and testing the specimens. Alison Parsons carried out data entry and cleaning, as well as providing invaluable office support.

\section{References}

1. Chlamydia - a testing issue. Lancet. 2005;365(9460):630.

2. Cook RL, Hutchison SL, Ostergaard L, Braithwaite RS, Ness RB. Systematic review: noninvasive testing for Chlamydia trachomatis and Neisseria gonorrhoeae. Ann Intern Med. 2005;142(11):914-25.

3. US Preventive Services Task Force. Screening for Chlamydial Infection: US Preventive Services Task Force Recommendation Statement. Rockville, MD: Agency for Healthcare Research and Quality; 2007. http://www.ahrq.gov/clinic/uspstf07/chlamydia/chlamydiars.ht.

4. Association of Public Health Laboratories. Laboratory diagnostic testing for Chlamydia trachomatis and Neisseria gonorrhoeae. Expert Consultation Meeting Summary Report; January 13-15, 2009; Atlanta, GA. Silver Spring, MD: Association of Public Health Laboratories; 2009. http://www.aphl.org/aphlprograms/infectious/std/ documents/ctgclabguidelinesmeetingreport.pdf.

5. Brooks D. The management of suspected urinary tract infection in general practice. Br J Gen Pract. 1990;40(339):399-401.

6. Foxman B. Epidemiology of urinary tract infections: incidence, morbidity, and economic costs. Am J Med. 2002;113(Suppl 1):5-13.

7. Johnson RE, Newhall WJ, Papp JR, et al. Screening tests to detect Chlamydia trachomatis and Neisseria gonorrhoeae infections - 2002. MMWR Recomm Rep. 2002;51(No. RR-15):1-38.

8. Centers for Disease Control and Prevention. Epi Info Version 3.5.1. 2008. http://www.cdc.gov/epiinfo/.

9. Diem K, ed. Scientific Tables 6th edition. Ardsley, New York: Geigy Pharmaceuticals; 1962.

10. Canterbury District Health Board. Specimen Collection Guidelines Mid-stream Urine (Female). http://www.cdhb.govt.nz/chlabs/patient/ pdfs/msu_female.pdf.

11. Ostergaard L, Moller, JK, Andersen B, Olesen F. Diagnosis of urogenital Chlamydia trachomatis infection in women based on mailed samples obtained at home: multipractice comparative study. BMJ. 1996;313:1186-1189.

12. Mangin D, Toop L, Chambers S, Ikram R, Harris B. Increased rates of trimethoprim resistance in uncomplicated urinary tract infection: cause for concern? N Z Med J. 2005;118(1225):U1726. 